\title{
HÁBITOS Y HABITUS EN LA TRANSFORMACIÓN CULTURAL: ESTUDIO DE UNA ORGANIZACIÓN DEL SECTOR ENERGÉTICO*
}

\author{
DAIMER HIGUITA LÓPEZ \\ UNIVERSIDAD NACIONAL DE COLOMBIA
}

Recibido/ Received/ Recebido: 14/01/2014 - Aceptado/ Accepted / Aprovado: 05/08/2014

\begin{abstract}
Resumen
Con base en el trabajo empírico desarrollado en una organización del sector energético y en las nociones de hábito en Geoffrey Hodgson y de habitus en Pierre Bourdieu, el presente artículo parte de la pregunta: ¿cuál es el rol que juegan los habitus de los trabajadores en la formación de hábitos que reflejan una transformación de la cultura organizacional? En esa dirección, se discute la afinidad entre los habitus de los trabajadores y la gestión cultural, como factor que potencia las disposiciones de estos y que contribuye con la formación de hábitos. Asimismo se trazan algunos elementos que participan en la configuración de hábitos que se constituyen en rasgos culturales en la organización.

Palabras clave: Gestión cultural, Habitus, Hábitos, Cultura organizacional.
\end{abstract}

\section{HABITS AND HABITUS ON THE CULTURAL TRANSFORMACION: A STUDY IN AN ORGANIZATION OF THE ENERGETIC SECTOR}

\begin{abstract}
In base of the empiric work developed in an organization of the energetic sector and the notions of habit in Geoffrey Hodgson and of habitus in Pierre Bourdieu, this article presents part of the question: What's the role that plays the workers habitus in the formation of habits that reflect a transformation of the organizational culture? In this direction, it's discussed the affinity between the workers habitus and the cultural development, as factor that strengthens the arrangements of these and contributes to the formation of habits. Also we trace some participant elements in the configuration of habits that constitute the cultural features of organization.
\end{abstract}

Keywords: Cultural Development, Habitus, Habits, Organizational Culture.

\footnotetext{
Artículo de investigación derivado del proyecto titulado "Los hábitos en los trabajadores generados por la gestión cultural y su incidencia en la cultura organizacional". Investigación realizada entre 2012 y 2013.

** Administrador de Empresas de la Universidad de Antioquia, Magister en Ciencias de la Administración de EAFIT. Profesor de la Escuela de Administración y Contaduría Pública, Universidad Nacional de Colombia. Miembro del grupo de investigación: Emprendimiento y Mipymes. Correo electrónico: dhiguital@unal.edu.co. Tel: (57) (1) 3165000 Ext. 12380. Dirección postal: Cll. 25 \# 40 - 74 , Facultad de Ciencias Económicas, Edificio 310, Universidad Nacional de Colombia, sede Bogotá.
} 


\title{
HÁBITOS E HABITUS NA TRANSFORMAÇÃO CULTURAL: UM ESTUDO EM UMA ORGANIZAÇÃO DO SETOR ENERGÉTICO
}

\begin{abstract}
Resumo
Com base no trabalho empírico desenvolvido em uma organização do setor energético e nas noções de hábito em Geoffrey Hodgson e de habitus em Pierre Bourdieu, o presente artigo parte da pergunta: qual é o papel dos habitus dos trabalhadores na formação de hábitos que refletem uma transformação da cultura organizacional? Nessa direção, discute-se a afinidade entre os habitus dos trabalhadores e o gerenciamento cultural, como fator que potencia as disposições destes e que contribui com a formação de hábitos. Também se traçam alguns elementos que participam na configuração de hábitos que se constituem em traços culturais da organização.
\end{abstract}

Palavras chave: Gerenciamento cultural, Habitus, Hábitos, Cultura organizacional.

Higuita, D. (2015). Hábitos y habitus en la transformación cultural: estudio de una organización del sector energético. En: Revista de la Facultad de Ciencias Económicas de la Universidad Militar Nueva Granada. rev.fac.cienc.econ, XXIII (1).

JEL: A14, M14.

\section{Introducción}

En 2005, el entonces gerente de la organización en estudio, inspirado en el caso Semco ${ }^{1}$, emprendió un programa para transformar la cultura organizacional. Ocho años después, tal transformación parece evidente. Por tanto, se dirá con Sainsaulieu (1997) que las organizaciones no son simples aparatos de producción. Ellas se han convertido en verdaderas instituciones sociales como la escuela o la iglesia, capaces de hacer evolucionar los comportamientos de los trabajadores y de la cultura.

Sin embargo, tanto Strategor (1995) como Emirbayer \& Johnson (2008) afirman que cuando un individuo llega a una empresa trae consigo unos antecedes, una historia, un habitus. Y el habitus se entiende como el conjunto de esquemas generativos a partir de los cuales los sujetos perciben el mundo y actúan en él. Y esos esquemas han sido conformados a lo largo de la historia de cada sujeto (Bourdieu, 2001).

Bourdieu (2000) afirma que cuando el habitus entra en relación con un mundo social del cual es produc- to, se encuentra como pez en el agua. Asimismo, el autor plantea que en determinadas situaciones se puede producir una "histéresis de habitus", en la cual las disposiciones aparecen inadecuadas a las condiciones presentes porque están ajustadas a otro tipo de condiciones.

Aunque pueden ser múltiples los factores que participan en la evolución de la cultura, el estudio que dio pie a estas líneas se focalizó en la generación de hábitos en los trabajadores, como una manera de comprender la transformación cultural. En consecuencia, este artículo se construye alrededor de la siguiente pregunta: ¿cuál es el rol que juegan los habitus de los trabajadores en la formación de hábitos que reflejen la transformación cultural de esta organización? La investigación se desarrolló con la metodología de estudio de caso en la organización que se denominará $\mathrm{PDE}$, la cual produce servicios de ingeniería.

Así, este texto se ha estructurado en cinco partes. En la primera se presenta la revisión de la literatura. En la segunda se describen las categorías preliminares en la investigación. Posteriormente, se delinea la me-

1 Semco es la empresa brasilera conocida por su modelo de gestión diferente al tradicional. 
todología empleada. En la cuarta parte se exponen los resultados y su discusión. El documento termina con las conclusiones sobre el papel de los habitus, los hábitos y la gestión en la cultura organizacional.

\section{Revisión de la literatura}

¿En qué se focalizan las investigaciones sobre el fenómeno cultural en la organización? La revisión de la literatura indica que algunas investigaciones se focalizan en relacionar la cultura con aspectos como el clima organizacional, el liderazgo o la productividad, mientras que otros se concentran en estudiar la cultura en sí misma. Entre las publicaciones que abordan la cultura en relación con otras categorías se encuentran los trabajos de Armenakis \& Wigand (2010) quienes estudian el impacto de los stakeholders en la cultura; Enríquez (2007), quien estudia la relación de la cultura con el aprendizaje organizacional; Gonzales \& Parra (2008), la relación con el clima, la motivación y el liderazgo; Montealegre \& Calderón (2007), la relación con el cambio; Sánchez et. al. (2007), con los equipos de trabajo; Song, Baek \& Chermack (2009), también con el aprendizaje.

En las investigaciones donde la cultura organizacional ha sido estudiada en sí misma, el propósito ha sido hacer una descripción del fenómeno como tal o plantear algunos modelos para su abordaje. Entre estos estudios están Aguilar (2006), quien propone una forma de diagnosticar la cultura; Barciela (2007), que se focaliza en cómo se gestiona; Calderón (1999), que analiza la concepción y el significado de cultura como categoría; García (2006), que aporta una concepción desde lo teórico; Kondra \& Hurst (2009), que estudian la transmisión de la cultura entre actores; Rodríguez (2010) que se concentra en las características de este fenómeno organizacional.

De otro lado, se evidencian varios estudios sobre la generación y formación de hábitos. Por ejemplo, Lally et al. (2011), muestran que el cambio en el comportamiento se experimenta inicialmente como un esfuerzo cognitivo, y que los hábitos se forman en contextos de trabajo. Hunt, (2010), expone cómo los hábitos influencian el ritmo de los investigadores en una investigación. Verplanken \& Wood (2006) expresan que las intervenciones efectivas para cambiar hábitos implican alterar factores ambientales que inducen o activan el desempeño del hábito. En tanto que Ikefuji \& Mino (2009) sostienen que además de las rutinas, la formación de hábitos está atravesada por un proceso individual. Para terminar, Hodgson (2007) sostiene que los mecanismos de replicación de los hábitos son incentivos, represión e imitación.

En cuanto a los habitus en la cultura organizacional, hay algunas aproximaciones en Voronov \& Vince (2012) quienes vinculan las emociones con el tema institucional; Ignatow (2009) quien se aproxima a la cultura como una cognición incorporada; Watts (2010) que estudia el papel de la identificación en la cultura. Ahora bien, desde una perspectiva sociológica se tienen los trabajos de Biggart \& Beamish (2003) quienes comparan el hábito con la práctica y la costumbre, y Mangi (2009) quien hace una crítica al neoinstitucionalismo. El trabajo de Ojeda (2009) es un ejemplo de la aplicación de los habitus a la cultura organizacional. Además, hay dos autores que se han convertido en puntos de referencia para aproximarse a la cultura organizacional desde los habitus. En primer lugar, Hallett (2003), quien a partir del paralelo entre las propuestas de Bourdieu y Goffman propone el orden negociado como una forma de construir cultura. El bien reconocido crítico Alvesson (1994), asocia la construcción de cultura a las lógicas de la identidad en las organizaciones.

\section{Categorías preliminares en la investigación}

Para Sainsaulieu (1997, 194), la empresa, al organizar los asuntos entre individuos en un conjunto de relaciones intensas, cognitivas y afectivas, es en realidad un lugar de alta socialización, de construcción de sí mismo y de representación del mundo. Agrega que, como la escuela, la familia o el barrio, la empresa es fuente de aprendizaje cultural. Por su parte, Gilbert et al. $(2005,162)$ sostienen que:

"Michel Crozier y otros investigadores han demostrado el rol de socialización jugado por la empresa. En una primera fase de la vida, los individuos son modelados por las instituciones primarias como el entorno familiar o la escuela. La cultura inicial 
constituye aquí un elemento que estructura la personalidad de cada uno. En otros contextos, hay un aprendizaje - en situación - de otros comportamientos, de nuevas maneras de hacer o de pensar. La empresa es concebida como una institución secundaria".

En otras palabras, la familia y la escuela son instituciones formadoras de hábitos y de disposiciones en el individuo (específicamente en el niño), a través de procesos de inculcación y de incorporación, como lo afirma Bourdieu (1982). Mientras que la organización se ha convertido en una institución secundaria. Es decir, la organización parece ser formadora de hábitos en el adulto. Y como se dijo, el trabajador llega a la organización con una historia incorporada, con su habitus.

Así entonces, la transformación cultural en la organización PDE se analizó desde los hábitos y el habitus. Así, en este apartado se expone, en primer lugar, la noción de hábitos desde Geoffrey Hodgson. Posteriormente, se esboza lo que se entiende por gestión cultural. Finalmente, se menciona la categoría habitus de Pierre Bourdieu.

El hábito es entendido como una disposición, propensión o capacidad adquirida. Para los institucionalistas, los hábitos tienen un papel esencial en el surgimiento y permanencia de las instituciones. Las instituciones, afirma Hodgson (2004), moldean hábitos y preferencias en los individuos y, a su vez, los individuos afectan las instituciones.

Para comprender el caso de esta organización, se introduce la noción de hábitos, entendidos como repertorios inconscientes de la conducta potencial, que pueden ser provocados o reforzados a partir de un contexto apropiado (Hodgson, 2007). En esta perspectiva, más que comportamientos, los hábitos son tendencias o propensiones hacia la acción. Una acción (o comportamiento) de un individuo no necesariamente es un hábito. Lo será en la medida en que dicho individuo posea la tendencia o disposición hacia esa acción (Hodgson, 2004).

En otras palabras, el hábito no es la acción (aunque se materialice en ésta) sino la tendencia hacia esa acción. En consecuencia, lo que diferenciaría el hábito de la conducta es que éste precede la acción, mientras que aquella refleja en sí misma una acción ${ }^{2}$. Por lo tanto, cuando un individuo tiene una disposición o hábito asociado a un rasgo cultural (como por ejemplo, la puntualidad) podría pensarse que la transformación cultural se concreta, al menos, en ese rasgo.

En la investigación que originó este artículo, la cultura se entendió como un conjunto ligado de maneras de pensar, de sentir y de actuar que, siendo aprendidas y comprendidas por una pluralidad de personas, sirven para construir a esas personas en una colectividad particular diferenciada (Rocher, citado por Aktouf, 1990). Y por tanto, la formación del hábito en el trabajador fue tomada en la investigación como una forma de aprehensión de la cultura. $\mathrm{O}$ dicho de otra forma, la transformación cultural tendría, entre otros efectos, una incidencia en la formación de hábitos en los individuos.

Por otro lado, sabemos que las organizaciones tienen unas políticas y que los directivos "instituyen" programas, reglas, rutinas, con el propósito de moldear una "cultura organizacional", pues como afirman Aubert \& Gaulejac (2007) se trata de hacer querer más que de hacer hacer, no se trata de humanizar las normas sino de normalizar al hombre.

De allí que se definió por gestión cultural todas aquellas prácticas, políticas, decisiones y acciones deliberadas de los directivos encaminadas a modificar la cultura. Dicha gestión se traduce, por un lado, en el diseño de manifestaciones culturales y, por el otro, en establecer los mecanismos organizacionales (Levin \& Gottlieb, 2009) que se utilizan para la difusión, transmisión y reforzamiento de dichas manifestaciones entre los trabajadores.

2 El diccionario de la Real Academia Española define estos conceptos de la siguiente manera. Conducta: Manera con que los hombres se comportan en su vida y acciones. (Psicol.): Conjunto de las acciones con que un ser vivo responde a una situación. Hábito: Modo especial de proceder o conducirse adquirido por repetición de actos iguales o semejantes, u originado por tendencias instintivas. Información extraída de: www.rae.es 
Las manifestaciones culturales son esas expresiones de la organización, tales como símbolos, rituales, valores, normas, rutinas, metáforas, eslóganes, historias, logos, lenguaje, que configuran la cultura y permiten diferenciar una organización de otra (Hofstede, 1999). Los mecanismos son dispositivos que los directivos utilizan para buscar que los trabajadores se apropien, aprehendan o se adapten a la cultura. Algunos de estos dispositivos tienen lugar desde el momento en que los nuevos empleados llegan a la empresa, como los programas de inducción, de capacitación, mientras que otros mecanismos obedecen a las prácticas de socialización en la organización.

Esas manifestaciones y esos mecanismos propuestos por la dirección de esta organización se enlistan en la Tabla 1.

Ahora bien, cuando una persona llega a trabajar a una empresa trae consigo un bagaje, una historia y su habitus, que funciona como un sistema de normas, más o menos conscientemente integrado. Así lo plantea Strategor al sostener que "cuando un individuo pasa a ser miembro de una organización, su habitus va a servirle de brújula interna, guiando sus acciones" (Strategor, 1995, 462). Por consiguiente, es importante recordar que en el estudio no se tomó al trabajador como un actor inerte o desprovisto de un pasado. Al contrario, se le vio como un trabajador con una historia incorporada y dotado de un habitus.

Por habitus Bourdieu \& Wacquant (1995) y Bourdieu (2000, 2007), entienden el conjunto de esquemas generativos a partir de los cuales los sujetos perciben el mundo y actúan en él. Estos esquemas generativos están socialmente estructurados: han sido conformados a lo largo de la historia de cada sujeto y suponen la interiorización del campo concreto de relaciones sociales en el que la persona se ha conformado como tal. Pero al mismo tiempo son estructurantes: son las estructuras a partir de las cuales se producen los pensamientos, percepciones y acciones del individuo. El habitus forma un conjunto de esquemas prácticos de percepción, apreciación y

Tabla 1. Elementos de la gestión cultural ${ }^{3}$

Factores asociados a la filosofía y al sistema cultural que se deseaba promover

- Calidad a tiempo.

- Importancia de las personas.

- Comunicación abierta y honesta

- Participación de empleados en decisiones

- $\quad$ Basado en confianza y respeto en la gente.

- $\quad$ Enfocados en entrega al cliente, con responsabilidad.

- Compromiso.

- $\quad$ Flexibilidad para trabajar.

- Trabajo en equipo.

- Autonomía.

Cambios en las formas de trabajar y algunos mecanismos que empezaron a implementarse

- $\quad 90 \%$ de los empleados con contratos a término indefinido.

- $\quad$ Programa de inducción.

- $\quad$ Libertad en el horario de trabajo.

- No hay procedimientos. Hay Guías que se usan como estándares operacionales

- Grupos de discusión para acompañar la transformación cultural

- Difusión de indicadores (comunicación, participación, satisfacción del cliente, medio ambiente).

- $\quad$ Las personas definen la fecha de sus vacaciones.

- $\quad$ Los empleados entrevistan sus futuros compañeros de trabajo.

- No hay evaluaciones anuales.

evaluación a partir de los cuales se generan las prácticas de las personas.

Bourdieu \& Wacquant (1995) presentan la génesis del habitus como proceso de inculcación de un arbitrario cultural y como incorporación de determinadas condiciones de existencia; lo que plantea dos modos distintos de generación del habitus: la inculcación y la incorporación. La inculcación supone una acción pedagógica efectuada dentro de un espacio institucional -familiar o escolar, y organizacional, como lo afirma Sainsaulieu (1997) - por actores dotados de autoridad. La incorporación, en cambio, remite a la idea de una interiorización por los sujetos de las regularidades inscritas en sus condiciones de existencia.

La incorporación de los habitus está relacionada con otro tema central en Bourdieu \& Wacquant (1995) y

3 Fuente: Elaboración propia a partir de: i) entrevista con el gerente; y ii) documentos proporcionados por el área de Talento y el área de Identidad y Compromiso. 
Bourdieu (2000, 2007): el "sentido práctico" o "racionalidad práctica". Con esta, Bourdieu critica las teorías de la "racionalidad" y plantea las diferentes racionalidades prácticas de los sujetos, que son función de sus esquemas de acción y percepción -de sus habitus- formados en unas condiciones sociales particulares.

En este sentido, la formación de hábitos no estaría desligada del conjunto de esquemas generativos a partir de los cuales los sujetos perciben el mundo y actúan en él. Esquemas conformados a lo largo de la historia de cada sujeto. Es decir que en la formación del hábito podría estar presente el habitus. Empero Hodgson (1996) como Sainsaulieu (1997) sostienen que la organización es formadora de hábitos y modeladora de comportamientos, es decir, que en la transformación cultural estaría presente la gestión de la cultura en la organización. De allí que algunas de las preguntas de la investigación fueron: ¿la transformación cultural supone la formación de hábitos en los trabajadores?, y en esa formación de hábitos ¿participan los habitus de los trabajadores y la gestión cultural?

Una vez esbozados estas categorías conceptuales, a continuación se delinea la metodología que se empleó para la producción de los datos.

\section{Metodología}

La recolección de información en la organización tuvo dos etapas. En la primera etapa, se buscaba identificar qué tenían en mente los directivos en términos de gestión cultural, es decir, qué deseaban transformar y cómo buscaban hacerlo. En esa etapa se hicieron entrevistas semi-estructuradas a 12 directivos y se examinaron diferentes documentos.

Algunos de los interrogantes de las entrevistas apuntaban a indagar por el diseño de manifestaciones culturales (su proceso y sus participantes) y por los mecanismos de gestión cultural. Las personas fueron seleccionadas por el rol que han tenido en la configuración de mecanismos culturales, por la información que poseían y por la disponibilidad para participar en el estudio. De otro lado, se analizaron documentos impresos y archivos donde se plantean algunas políticas de transformación cultural. También se abordaron documentos donde se hace seguimiento a la organización en términos de indicadores de gestión.

En la segunda etapa, se exploraron qué hábitos fueron formándose a partir de los cambios propuestos (expresados en la Tabla 1) y el rol que han jugado los habitus y la gestión cultural en la formación de hábitos en los trabajadores. En esta etapa se aplicó una encuesta, se efectuaron grupos foco, entrevistas en profundidad a trabajadores y visitas de observación en la organización.

Dado el tamaño de la organización, se consideró que la encuesta era un instrumento adecuado para obtener gran cantidad de información de los trabajadores. La encuesta incluyó tanto preguntas abiertas como cerradas y tenía como propósito identificar: i) cuáles son las manifestaciones culturales y los mecanismos de gestión cultural a los que se les atribuye mayor importancia por parte de los trabajadores; ii) hábitos asociados a valores, mecanismos, manifestaciones y filosofía organizacional. En total 199 personas respondieron la encuesta. Esta fue aplicada online por recomendación de los mismos trabajadores durante la prueba piloto.

El grupo foco permitió que los mismos participantes pudieran profundizar en la información que se había recolectado con la encuesta. En total se realizaron nueve grupos foco. Cada grupo estuvo conformado en promedio por siete personas. Los propósitos del grupo foco fueron: producir nuevas significaciones a partir del discurso, corroborar los primeros hábitos en los trabajadores, analizar la participación de los mecanismos de gestión cultural y validar que las personas determinaran si habían adquirido algún hábito que otros también habían adquirido.

Para Bonilla \& Rodríguez $(2005,134)$ "la muestra no se selecciona, se configura, es decir, se va estructurando a través de las diferentes etapas del proceso de recolección de datos". Así que esta fue la dinámica que siguió la aplicación de las 12 entrevistas en profundidad realizadas con trabajadores, las cuales se acompañaron de una carta asociativa. Todas las entrevistas se aplicaron en las instalaciones de la 
compañía y tenían como objetivo rastrear el rol del habitus en la transformación cultural.

Por su parte, la observación se aplicó en paralelo al uso de las demás técnicas descritas acá. Se realizaron siete visitas a la organización con propósitos de observación. La utilización de la observación durante la investigación fue de gran utilidad porque permitió contrastar la información que se fue recolectando con otras técnicas. Todo el trabajo de campo se desarrolló durante 14 meses.

Respecto al análisis, se acudió al análisis de contenido, entendido como un método de codificación bajo diversas categorías resaltando sus características con miras a comprender el sentido exacto y preciso del mensaje (Zapata \& Rodríguez, 2008, 46). En cuanto al uso de software se utilizó el SPSS 20 y el MaxQda 10 para el análisis de la información recolectada con la encuesta. Como un paso previo a la categorización, se construyeron redes conceptuales (Alvesson \& Karreman, 2011) a partir de los distintos elementos que emergieron del análisis de la información.

A partir de la identificación de los hábitos que se asocian a medidas implementadas en la gestión cultural, como la libertad en el horario o la autonomía para trabajar, se les preguntó a los participantes qué aspectos habían incidido en que ellos adquirieran un determinado hábito. Esas respuestas se agruparon en dos categorías: aquellos aspectos relacionados con la especificidad del individuo (sus habitus) y aquellos elementos asociados al contexto organizacional, es decir, a lo que encuentran en la organización. Este análisis permitió rastrear los mecanismos que, de alguna manera, participan en la formación de hábitos. A continuación se exponen los hallazgos, indicando que las expresiones de los informantes se han puesto entre comillas (" ").

\section{Resultados y discusión}

Después de varios años de iniciados los cambios, los trabajadores de esta organización han adquirido algunos hábitos y disposiciones que evidencian la transformación cultural. Los hábitos, entendidos como propensiones hacia la acción, se materializan en comportamientos. El que esta empresa se caracterice hoy por ofrecer servicios con calidad a tiempo es posible porque las personas tienen la disposición de participar en las decisiones y procesos, y lo hacen; porque poseen la disposición a comprometerse en los proyectos que se les encomiendan, y lo hacen; porque tienen la disposición a desempeñarse con responsabilidad, y lo hacen. Porque tienen la disposición a actuar con autonomía para desarrollar su labor. Estas particularidades pudieron evidenciarse en el discurso de los informantes, en la descripción de la cultura de trabajo que hacen las mismas personas y en la observación efectuada en la organización. Algunos de esos hábitos se enlistan en la Tabla 2.

Tabla 2. Algunos hábitos adquiridos por los trabajadores ${ }^{4}$

\begin{tabular}{|c|c|}
\hline & \\
\hline $\begin{array}{ll}\text { - } & \text { Hacer el trabajo siguiendo las } \\
\text { - } & \text { Guías pero con flexibilidad*. } \\
\text { - } & \text { Trabajar en equipo. * } \\
\text { - } & \text { otractuar áreas. } \\
\text { Participar en reuniones con } \\
\text { - } \quad \text { grupos de trabajo.* } \\
\text { Hacer seguimiento a los pro- } \\
\text { - yectos. } \\
\text { - } \quad \text { Revisar pendientes. } \\
\text { - } \quad \text { Ajustar planes de actividades. } \\
\text { Desempeñarse con respon- } \\
\text { sabilidad. }\end{array}$ & $\begin{array}{ll}\text { - } & \text { Tratar a los demás con res- } \\
\text { - } & \text { peto. }{ }^{*} \\
\text { - } & \text { dad. }{ }^{*} \\
\text { - } & \text { Participar en decisiones. }{ }^{*} \\
\text { - } & \text { Considerar en los proyectos } \\
\text { - } & \text { las exigencias del cliente. } \\
\text { Desarrollar la labor con auto- } \\
\text { - } \quad \text { nomía. }{ }^{*} \\
\text { - } \quad \text { Asumfiar en los otros. * } \\
\text { - } \quad \text { cer bien las cosas. }{ }^{*} \\
\text { Hacer una pausa para conver- } \\
\text { sar con algunos compañeros. }\end{array}$ \\
\hline
\end{tabular}

En este punto conviene hacer una precisión. No todos los hábitos que los trabajadores adquieren en la organización se derivan directamente de la gestión cultural. Los hábitos que han sido marcados con un asterisco (*) son hábitos que se asocian con los elementos de la gestión cultural, planteados en la tabla 1. Los otros son hábitos a los cuales no se les encontró algún vínculo con los elementos de la gestión cultural.

Pero ¿cuál es el rol que juegan los habitus de los trabajadores en la formación de esos hábitos?

4 Fuente: Elaboración propia. 
"Pensar en términos de habitus será pensar la acción social en términos de disposiciones, y estas no son potencia pasiva, sino un primer grado de determinación que orienta la acción, pero no son tampoco acción, por lo tanto suponen inclinación pero no movimiento. Decir que el individuo está dispuesto no es decir que actuará mecánicamente de un determinado modo, (...) decir que tiene un habitus es decir que se inclina a actuar a partir de unos haberes, que son saberes, sentimientos, preferencias acumuladas en experiencias anteriores. Estar dispuesto así a la acción no es estar determinado a ella, sino que queda abierto siempre el margen de un acto segundo que actualice lo que está siempre en potencia" (Martínez, 2007, 81).

A partir de los hábitos de la tabla anterior, se configuraron dos situaciones: i) donde hay una afinidad entre la gestión cultural y los habitus, y las disposiciones de las personas se ven potencializadas; ii) donde no hay tal afinidad y se generan nuevas disposiciones.

El primer caso está conformado por aquellas situaciones donde hay una afinidad entre elementos de la gestión cultural y los habitus de las personas. Veamos un ejemplo con el valor corporativo Respeto. Para varios de los informantes, el respeto hace parte de las disposiciones del empleado, configuradas por sus experiencias. Y esas disposiciones que ellos ya tenían al momento de ingresar a trabajar es lo que ha conducido a que dicha manifestación promulgada por la gestión cultural sea, para ellos, muy compatible. Allí, lo que hace la gestión cultural es disparar o potenciar las disposiciones que los trabajadores traen producto de su historia personal. Por esta razón, cuando se habla de los rasgos culturales (en términos de formas de trabajar o de relacionarse con los otros), los informantes sostienen que hay "cosas que se traen desde la casa".

Bourdieu \& Wacquant (1995) sostiene que cuando el habitus entra en contacto con un mundo social del cual es producto, se encuentra como pez en el agua y el mundo le parece autoevidente. En nuestro caso, se encontró que cuando una manifestación cultural particular de la organización está cerca de las disposiciones configuradas por experiencias e historia personal del trabajador, dicha manifestación cultural le es, en algún sentido, más familiar. Esto se observó, por ejemplo, en las personas que se formaron en ambientes participativos y se hallaron en una organización que fomenta la participación. Con "formó" se hace referencia a los esquemas mentales que, de acuerdo con ellos mismos, las personas recibieron en la familia y en su entorno social. Esta situación también se encontró en el caso de valores como el respeto o la responsabilidad y en el caso de disposiciones que traían las personas como la de ser autónomos o de confiar en el otro.

En el segundo caso se observó que, en algunas situaciones, no hay una afinidad entre los habitus de las personas y elementos de la gestión cultural. Es decir, los habitus se encuentran con una estructura social (ofrecida por el sistema cultural de la organización) diferente a las condiciones en las cuales éstos se conformaron. Por ejemplo, las personas que no tenían la tendencia a ser participativos y se encontraron con un ambiente que fomenta la participación. En este caso, las personas realizan una valoración de los elementos $y$ rasgos culturales que afrontan, y les encuentran una cierta utilidad gracias a sus propios habitus, pues dijimos que estos son esquemas de evaluación. Y esta situación favorece la aparición o adopción de una nueva disposición. Un ejemplo de ello es el hábito de participar y aportar en reuniones. Esto se observó en las personas que decían ser muy poco participativas, que no tenían esa tendencia, pero que a partir de sus esquemas de percepción y valoración fueron adquiriendo ese hábito en esta organización.

Hasta aquí, es posible plantear que los habitus de las personas juegan un papel especial en la transformación de esa cultura, en la medida en que ayudan a potenciar o a generar nuevas disposiciones. Asimismo, esa valoración que hacen los individuos de lo nuevo y diferente que están afrontando (como la libertad en el horario) está permeada por las estructuras a partir de las cuales se producen las percepciones y acciones del trabajador.

Al observar qué cosas precisas inciden en que los trabajadores adquieran un determinado hábito, en el 
análisis de la información emergieron siete elementos. Estos elementos se han clasificado en dos grupos: i) aquellos factores que dependen del individuo $y$ de sus esquemas de percepción y evaluación y ii) la realidad exterior que existe en la organización independiente de un sólo individuo. En otras palabras, sin un solo trabajador se va de la organización, estos factores siguen allí, o cuando un nuevo empleado ingresa a esta empresa, se encuentra con estos elementos $^{5}$. El sentido, la identificación y la satisfacción personal son factores que dependen del individuo. Es decir, de la individualidad de cada trabajador. Procesos cotidianos y control social son elementos que hacen parte de la realidad exterior al sujeto. Es decir, son situaciones que existen en la organización, independientemente del trabajador. Adicionalmente, hay dos factores que parecen pertenecer a ambas circunstancias (el individuo y la realidad exterior). Esos factores son ambiente de trabajo y compenetración con el grupo. Muy probablemente estos siete no son los únicos elementos presentes en la formación de hábitos, pero si son los que emergieron en el análisis de la información en esta organización.

\subsection{Sentido}

Las personas afirman que han adquirido un determinado hábito porque le encuentran algún sentido a dicho hábito en la organización o porque creen que haciendo las cosas de esa manera, las cosas funcionan. Este sentido está ligado a formas específicas de trabajar, de proceder o de organizarse en términos de la evaluación, coordinación o ejecución de los proyectos. El sentido acá es tomado en función de los resultados que se obtienen o de una utilidad al proceder de determinada manera, como por ejemplo, al revisar pendientes. Pero ese sentido que se le otorga a un hábito es dado por cada sujeto. Es decir, cada trabajador es quien elabora una apreciación de "esa" manera de proceder, como por ejemplo, hacer el trabajo siguiendo unas guías.

Ahora bien, como se dijo páginas atrás, el habitus forma un conjunto de esquemas prácticos de percepción, apreciación y evaluación a partir de los cuales se generan las prácticas de las personas. Un ejemplo de esto se encuentra en el hábito que han adquirido los trabajadores de desarrollar su labor con autonomía. Y aquí conviene hacer una precisión. Aunque el habitus se configura a partir de las estructuras sociales, implicando la incorporación de lo social en el individuo, este habitus aflora, emerge, en las acciones que emprende el individuo. En este caso, desarrollar la labor con autonomía es algo a lo que los trabajadores le encuentran sentido. En este momento, ese es un rasgo de la cultura en esta organización. Así como la participación o el compromiso. Y allí está presente el habitus pues es gracias a los esquemas prácticos de percepción que los trabajadores encuentran sentido a esas formas de comportarse o de trabajar.

Pero ¿por qué encontrar sentido participa en la formación de hábitos? Porque, tal vez, hacer las cosas de determinada manera (o hacer determinadas cosas, como revisar pendientes) no crea ningún choque en el empleado con sus propios esquemas de pensar y actuar. En esta medida, no es algo que las personas hacen contra su manera natural de proceder. Podría decirse que el sentido se vincula con lo que Bourdieu (2001) llama la racionalidad práctica. Ahora bien, refiriéndose a la construcción de la decisión para la acción, De la Garza Toledo $(2005,21)$ argumenta que hay dos modalidades:

"La de las prácticas reflexivas y la de las rutinarias. En ambas influyen en esa construcción presiones de las estructuras. (...) Estas presiones estructurales para convertirse en acción pasan mediante el aparato subjetivo de dar sentido. La decisión para la acción implica no solo pasar por un cálculo de optimización sino, de manera más amplia, por la constitución de configuraciones para dar sentido con componentes cognitivos, valorativos, estéticos, argumentativos".

Probablemente dicha apreciación para dar sentido es un proceso espontáneo, sin mucho cálculo. Pero cuando la persona reflexiona sobre el hábito adquiri-

5 En este caso se hace referencia a la realidad exterior vinculada con la organización y no con la sociedad. 
do, afirma encontrarle un sentido, una razón de ser, una funcionalidad. Esa forma de proceder o de trabajar (entiéndase ese hábito) tiene sentido para él. Ahora bien, esta apreciación no se da sólo frente a lo propuesto por la gerencia, sino también para lo que los trabajadores proponen. Es decir, en algunos casos son ellos los que encontrándole sentido a algo, lo proponen, como por ejemplo, ajustar planes de actividades $^{6}$.

\subsection{Procesos cotidianos}

En este caso, lo que ha favorecido la formación del hábito es la manera como se realizan los procesos cotidianamente en la organización. Es decir, las formas de trabajar implican unas guías, unos estándares y unas rutinas en las formas de proceder. Los participantes sostienen que el hecho de que diariamente deben hacer una determinada tarea para desarrollar su trabajo (como por ejemplo, hacer seguimiento a los proyectos), ha incidido en adquirir ese hábito. Aquí se observa ese elemento propuesto por Nelson \& Winter (2002), y también por Hodgson (2007), cuando hacen referencia a las rutinas organizacionales.

Así entonces, este elemento está asociado a la realidad exterior al individuo. Es una característica que hace parte de la organización y de su especificidad. Dado que esta es una organización que vende servicios, específicamente proyectos de ingeniería, existen un conjunto de protocolos de seguridad, de calidad, de mantenimiento de normas ambientales que conducen a la necesidad de tener unas guías para operar. Se trata, en términos de la gerencia, de que las personas sigan esas guías y no procedimientos rígidos. Sin embargo, los trabajadores saben que los protocolos y Guías son como herramientas que no deben olvidar. Y que están tan atentos a seguirlas y las siguen con tanta cotidianidad que llegan, en palabras de ellos mismos, "a interiorizar el proceso, a incorporar la guía".

Al respecto, Hodgson $(2004,13)$ sostiene que:
"Todo mundo acepta que la existencia de las instituciones depende de los individuos y que, en ocasiones, los individuos pueden cambiar las instituciones. Provoca mayor controversia que las instituciones, al estructurar, restringir y permitir comportamientos individuales, tienen el poder de moldear las disposiciones y comportamientos de los individuos. El hábito es el mecanismo clave en esa transformación".

De allí que esos procesos cotidianos se constituyan en una manera en que la organización moldea ciertos hábitos en los trabajadores. Por consiguiente, esos procesos cotidianos componen el segundo factor presente en la formación de hábitos.

\subsection{Ambiente de trabajo}

En el análisis de los datos emerge la idea de que el ambiente de trabajo conduce a las personas a la adquisición de ciertos hábitos. En este caso, las personas hacen referencia a la forma como se trabaja, pero no en términos de procedimientos (como en el caso anterior) sino de comportamientos y de relaciones con sus colegas ${ }^{7}$. Es decir, las personas van adquiriendo ciertos hábitos (como participar durante reuniones) en la interacción con los otros. O en formas de relacionarse que "cobijan" la operación diaria en la organización.

La interacción social puede entenderse como "la influencia recíproca de un individuo sobre las acciones del otro cuando se encuentran ambos en presencia física inmediata" (Goffman, 2001, 27). Para los trabajadores, las formas de comportarse, de interactuar y de relacionarse constituyen el Ambiente de trabajo, del cual cada uno se va impregnando en la cotidianidad. Y se va impregnando gracias al lenguaje, pues como sostiene un informante: "es que desde el primer momento uno escucha las personas hablar de autonomía". Pero además de la referencia al lenguaje, en la interacción está la vivencia, cuando agregan: "uno ve que las personas se tratan con respeto".

Este es uno de los hábitos que no se lograron vincular con la gestión cultural.

Esta es la razón por la cual los hábitos han sido agrupados en dos columnas en la Tabla 2. 
Ahora bien, quien habla de autonomía está tomando una posición (Emirbayer \& Johnson, 2008), lo mismo que aquel trabajador que se va impregnado de ese ambiente de trabajo.

En principio podría pensarse que este aspecto (ambiente de trabajo) hace parte de la realidad exterior al sujeto. En otras palabras, que así es como la organización opera independientemente de los trabajadores. Sin embargo, los individuos también están incluidos allí porque son ellos los que perciben. Recuérdese que en la interacción cotidiana, Goffman (2001) afirma que cada persona llega con sus propios pertrechos al encuentro con el otro, o podría decirse, en palabras de Lastra \& de la Rosa (2006), que se observa una consonancia entre lo psicológico y lo social. Pues son ellos, los trabajadores, los que sienten que ese es el ambiente (en términos de comportamientos y de relaciones con los otros) o que sencillamente "así se hacen las cosas". A partir de sus habitus, cada uno percibe esa forma específica de comportarse. Por lo tanto, aunque si hay una realidad exterior, esta queda "viciada" por la percepción individual. Por esta razón, se ha considerado que este elemento (ambiente de trabajo) hace parte de esas dos esferas: el individuo y la realidad exterior.

\subsection{Identificación}

En este caso, los trabajadores fueron enfáticos en sostener que ellos han adquirido ciertos hábitos porque "yo como persona me siento identificado con dicho hábito. Siempre he sido un convencido de eso". Aquí las personas hacen referencia a su historia personal y a diferentes experiencias que los han formado a lo largo del tiempo. En términos generales, la identificación puede entenderse como un mecanismo psíquico consciente o inconsciente que induce a un sujeto a comportarse, pensar, vincularse y sentir como otro que actúa como su modelo o encuentra en él afinidad en su comportamiento y el propio. Para Freud (1981, 2587) la identificación es conocida "como la manifestación más temprana de un enlace afectivo a otra persona" y "puede surgir siempre que el sujeto descubre en sí un rasgo común con otra persona".

En el caso de la identificación como factor que participa en la formación de hábitos se observan, sobre todo, hábitos relacionados con valores como el respeto y la responsabilidad. Por ejemplo, fue bastante reiterativo decir que la responsabilidad o el respeto "son cosas que se traen desde la casa". Este caso concreto se ve en el hábito (como disposición) de tratar al otro con respeto. Cuando las personas dicen que fue en su casa donde aprendieron esto, estamos frente al habitus que la persona ya trae al momento de ingresar a la organización. Ese habitus lo conduce a identificarse con ese elemento que fomenta la gestión cultural, que en este caso es un valor corporativo. De esta forma, el trabajador se identifica con la manifestación cultural y el entorno que afronta, donde se fomenta el respeto, potencia su disposición. Ahora bien, cuando las personas afirman que siempre se han identificado con el respeto, y encuentran una organización que fomenta el respeto, sienten que tienen un lugar en esa organización. O, en palabras de Watts (2010), encuentran una identificación con un aspecto específico o cultural de la organización.

Esta situación indicaría la presencia del habitus en ese proceso de formación de hábitos. Pero no se trata de una identificación del trabajador hacia la empresa en general, sino con ese aspecto particular que la gestión cultural plantea. Por ejemplo, ante la pregunta abierta: describa la filosofía de la organización, se observa que las personas hacen alusión fundamentalmente a los valores corporativos, es decir, los trabajadores definen la empresa como una organización de respeto o de confianza.

Las personas se sienten identificadas con algunos elementos de los valores (confianza, respeto) y de la filosofía (participación) porque, según ellos, es algo de lo que siempre han estado convencidos. Y, ¿qué quiere decir siempre?, ¿̇hace esto referencia a un individuo con historia?, ¿a un individuo con unas estructuras mentales que se han conformado en su interacción con un entorno social?, ¿estará allí su habitus a través de un esquema de evaluación al sostener "me siento identificado con dicho hábito"?

Así entonces, puede pensarse que esa Identificación (como elemento que participa en el proceso de formación de hábitos) está anclada a los valores que cubren las relaciones con el otro. En esta dirección, 
Sainsaulieu (1997, 198) sostiene que una escena social de la transformación cultural "es aquella del grupo donde el individuo vive relaciones durables $y$ diferentes de la vida cotidiana. Un espacio transicional, de acuerdo con el psicoanalista Winnicott, donde pueden operar desplazamientos, intercambios, juegos de identificación, de argumentación, de proyección de sí mismo sobre los otros, que ayudan a soportar el cambio de representaciones".

\subsection{Satisfacción personal}

Es bien conocida en la literatura sobre gestión humana, la noción de satisfacción laboral. Si se remite al concepto clásico, se encuentra que Herzberg, Mausner \& Snyderman (1960) planteó que la satisfacción laboral estaba determinada por factores motivacionales (asociados directamente con la tarea, con lo que hay que hacer) y factores de higiene (referidos a lo que se tiene en el trabajo). En esta organización se encuentra una situación particular que se ha querido denominar tal como los ingenieros la llaman: Satisfacción personal. No se refiere a la satisfacción laboral por lo que se hace sino a esa satisfacción por ser reconocido como ser humano, por sentir que se tiene un valor como persona, que es cada uno como trabajador el que cuenta. Las personas manifiestan que ser parte de esta empresa les genera satisfacción personal; y al adquirir, por ejemplo, el hábito de confiar en el otro o de participar en decisiones, sienten que esa satisfacción se mantiene o mejora.

¿Por qué podría pasar esto? Porque, probablemente, más que hacer (factores motivacionales) o tener (factores higiénicos), para las personas también cuenta el sentir, el ser y su realidad afectiva. Cuentan esos aspectos y momentos menos materiales donde las personas sienten que pueden expresarse y que su realidad afectiva también está presente. Al respecto, Smollan \& Sayer (2009) rastrean la importancia de aspectos afectivos, como las emociones, en los comportamientos y en la configuración de la cultura organizacional.

Adicionalmente, las personas sienten una satisfacción al sentir que se definen a sí mismos como responsables o comprometidos y que son vistos por los otros como responsables. Claro está que desde una perspectiva crítica se argumenta que en las organizaciones se busca mejorar la productividad con programas de formación que introducen el mensaje de la dirección en los empleados (Aubert \& Gaulejac, 2007). Por lo tanto, tal vez esta identificación sea manipulada por programas de formación o de satisfacción laboral. Pero lo que aquí ha importado es que la satisfacción personal que sienten los trabajadores ha participado en la adquisición de ciertos hábitos como actuar con responsabilidad o confiar en el otro. Y la responsabilidad o la confianza son rasgos de la cultura actual. Por lo que, posiblemente, esa satisfacción personal se potencia al actuar de determinada forma.

Ahora bien, esta situación no se encontró en todos los trabajadores, sino en aquellos con un habitus que los inclina a confiar en el otro, lo que permite observar la presencia del habitus ya que esta situación depende de cada individuo. De lo que aprecia, de lo que valora, de su propio sistema de disposiciones y su historia incorporada. Por ejemplo, al analizar las respuestas que estos informantes dan, cuando hablan de la filosofía de la organización, se encuentra que para ellos, esta es una organización que se confía en el empleado y que lo valora.

\subsection{Compenetración con el grupo}

En algunas ocasiones, las personas adquieren hábitos porque encuentran que esa es una manera de compenetrarse con el grupo de trabajo y de adaptarse a la organización. Se trata de un factor que pertenece tanto a la realidad exterior como al individuo. Las personas afirman: "es que si yo no trabajo con cumplimiento y compromiso me voy quedando atrás. No encajo en ese proyecto ni en esta empresa". En este caso, los individuos observan los rasgos del grupo (realidad exterior) pero son ellos mismos quienes sienten la necesidad de integrarse o sentirse parte de. ¿Y por qué esta situación entra en juego en la formación de hábitos? Al parecer, es un asunto que obedece a una necesidad humana de tener validación o legitimación (Bietti, 2009) por parte de los otros. En otras palabras, es un juego permanente entre el individuo y el grupo, donde a través de intercambios verbales e interacciones el primero se aproxima al segundo. En términos institucionales 
(Kondra \& Hurst, 2009), podría hablarse de un isomorfismo, de una imitación. No obstante, parece ser que esta situación va más allá de la imitación, en busca de un acople, de una compenetración con el grupo.

Pero también es un asunto de contrastación con lo que cada uno aporta y lo que imagina que los demás esperan de él, como en el caso del compromiso o la responsabilidad. Es decir, ser responsable con los deberes es algo con lo que me podría sentir bien, pero también es algo que sé que los demás esperan de mí. Y no ser responsable me impide "encajar en este proyecto o en esta empresa".

Al respecto, Hodgson (2004) sostiene que los hábitos de varios individuos los sostendrán de forma mutua en una estructura interconectada de comportamientos individuales recíprocos. Juntos estos comportamientos toman las cualidades colectivas asociadas con equipos. Tanto individuos como estructuras están implicados en todo el proceso (Hodgson, 2004). Y como se mencionó páginas atrás, aunque el habitus se configura a partir de la incorporación de lo social en el individuo, emerge en las acciones que este emprende. Por tal razón se habla del habitus como esquemas generadores y clasificadores de prácticas.

\subsection{Control social}

A diferencia de la compenetración con el grupo, el control social es un proceso más explícito para las personas. El control social hace parte de la realidad exterior. Las personas son conscientes de que hay unas "normas" que es necesario seguir. En el caso de la necesidad de compenetrarse con el grupo esta no parece ser siempre un asunto consciente. En el caso de las normas, son más conscientes porque lo viven. Los trabajadores se dan cuenta que algunos compañeros o colegas inducen a otras personas a hacer las cosas de cierta manera. Y en algunos casos, otros compañeros lo sugieren directamente, como en el hábito de ajustar planes de actividades.

En la teoría administrativa se habla de control social desde el trabajo de Elton Mayo (2003). En aquel caso, se afirmaba que los grupos incidían en que la producción individual no estuviera ni por encima ni por debajo de la media del grupo. Aquí se ha creado un contexto de control social para el mantenimiento de ciertas prácticas de trabajo como la libertad en el horario o el seguimiento de estándares operacionales. Por ejemplo, en el caso de la libertad de horario, los participantes afirman que es una situación donde hay personas que abusan de esta posibilidad. Es decir que hay personas que a veces no llegan cumplidas a una reunión con el argumento de que en la organización no hay horario. Pero ellos mismos se encargan de decirle al incumplido que, de un lado, es parte del respeto hacia los otros, llegar puntual, y del otro, además de la libertad en el horario, la filosofía de la empresa proclama el cumplimiento con el cliente y la calidad a tiempo.

Este control social ha sido auspiciado tanto por la gerencia como por los mecanismos que hacen acompañamiento a la gestión cultural. ¿Cómo lo hacen? en la inducción y en otras instancias de socialización, se les recuerda explícitamente a las personas que "la cultura se construye entre todos" y que ellos mismos pueden favorecer esta construcción, indicándole a sus compañeros cuando vean que estos no están "en sintonía con la cultura”.

\subsection{Atención}

Aunque aquí se hayan propuesto ejemplos para factores que contribuyen (o participan) en la configuración de hábitos, esto no quiere decir que exista una asociación uno a uno entre los hábitos y los factores. Es decir, un mismo factor puede participar en que se formen varios hábitos y, a su vez, la formación de un hábito puede estar influenciada por diferentes factores. Inclusive, el mismo habitus (como esquemas de percepción, apreciación y evaluación) está asociado a diferentes acciones en diferentes circunstancias (Gartman, 2007). El estudio de cuáles factores se articulan con qué tipo de disposiciones escapaban a los objetivos de la investigación. También escapan a los objetivos del estudio la cuantificación para decir, por ejemplo, que el control social incide el doble que la identificación.

Lo particular aquí es considerar (Emirbayer \& Johnson, 2008), que cada miembro de la organización trae sus habitus formados bajo condiciones específi- 
cas del pasado, y algunos de estos serán compartidos con otros miembros y algunos diferirán de los otros. Tal situación se observa en factores como ambiente de trabajo y compenetración con el grupo. Asimismo, ver que las interacciones modelan parcialmente las subjetividades, y los individuos, dotados de esquemas prácticos de acción, de un habitus (Mangi, 2009), van dando forma a esas interacciones y se van constituyendo en artífices de dinámicas organizacionales.

En la Tabla 3 se presentan estos factores, de acuerdo con la clasificación en términos de si son factores vinculados al individuo (a su habitus) o a la realidad exterior. En el primer caso, los informantes argumentan que su adaptación a los nuevos rasgos culturales se debe en parte a sus propios esquemas mentales (Biggart \& Beamish, 2003), conformados durante su historia personal. En el segundo caso, a lo que encuentran en la organización. Tal vez por esas experiencias anteriores, por el habitus de cada trabajador, fue común escuchar expresiones como "me identifico con ello", "siempre he sido un convencido", "es algo que se trae desde la casa", "siento que las cosas funcionan". En otras palabras, parte del porqué un trabajador se incorpora fácilmente a una cultura organizacional estaría influenciado por sus habitus.

Tabla 3. Factores que contribuyen con la formación del hábito ${ }^{8}$

\begin{tabular}{|c|c|}
\hline $\begin{array}{c}\text { Factores asociados al } \\
\text { habitus }\end{array}$ & $\begin{array}{c}\text { Factores asociados al } \\
\text { contexto exterior }\end{array}$ \\
\hline Sentido & Procesos cotidianos \\
\hline Identificación & Control social \\
\hline Satisfacción personal & \\
\hline
\end{tabular}

Finalmente, se dirá que la gestión cultural en esta organización se materializa en mecanismos como grupos de discusión, programa de inducción, política de participación, medidas como la autonomía y la flexibilidad para trabajar. Estos mecanismos participan en la formación de hábitos a través del impacto que logran tener sobre los factores que inciden en dicha formación. Así, el programa de inducción par- ticipa en identificación y control social; los grupos de discusión contribuyen con ambiente de trabajo, compenetración con el grupo, control social; la participación genera Satisfacción personal y ayuda al ambiente de trabajo; la flexibilidad y la autonomía impactan en aspectos como sentido, identificación, ambiente de trabajo, compenetración con el grupo y procesos cotidianos.

\section{Conclusiones}

En la formación de hábitos participan no solo elementos externos al individuo (que en algunos casos podría asociarse a la gestión cultural) sino también elementos que hacen parte de la interioridad y la especificidad de los trabajadores. Los hábitos que los trabajadores adquieren en la organización son una forma de transformación de cultura organizacional y los habitus son importantes tanto para la comprensión de "esa" transformación como para la formación de dichos hábitos.

La presencia del habitus permitiría decir que no es la gestión deliberada, unidireccional la que determina la cultura. Sin embargo, tampoco es un asunto exclusivo de la interacción social, puesto que en algunos casos, el sistema cultural 'potencia' en cada individuo lo que éste ya trae. En otras palabras, puede haber muchas interacciones, pero siempre hay un proceso individual de percepción. Una percepción atada a la historia personal.

Ahora bien, la gestión cultural a través de mecanismos (como el programa de inducción y los grupos de discusión) y de manifestaciones (como los valores y la filosofía organizacional) participa en el proceso de configuración o aprehensión de rasgos culturales, materializados en hábitos. ¿Por qué puede decirse que participa? Porque hay, efectivamente, algunos hábitos que son adquiridos por los trabajadores como el de participar.

Si bien comúnmente se da el rol principal en la transformación de la cultura al líder, la investigación permite pensar que dicha transformación no es exacta-

8 Fuente: Elaboración propia. 
mente a imagen y semejanza del directivo, pues la historia y los antecedentes de los trabajadores tienen una importancia en este proceso que no se puede desconocer. De manera similar, se pudo observar que la gestión cultural si tiene un impacto sobre la cultura, pero una vez más, los habitus juegan un papel fundamental en la manera como las personas perciben y evalúan dicha gestión cultural.

\section{Referencias}

Alvesson, M. (1994). Talking in organizations: managing identity and impressions in an advertising agency. En: Organizations studies, 15(4).

Alvesson, M. \& Karreman, D. (2011). Qualitative research and theory development. London: SAGE.

Aguilar, A. (2006) El diagnóstico de "la cultura organizacional o las culturas de la cultura". En: Global media Journal, 3(6).

Aktouf, O. (1990) El simbolismo y la cultura organizacional. De los abusos conceptuales a las lecciones de campo. Quebec: PulEska. Traducción al español. En: AD MINISTER, Universidad EAFIT, (1): 65-94.

Armenakis, A. \& Wigand, J. (2010) Stakeholder actions and their impact on the organizational cultures of two tobacco companies. En: Business and Society Review, Center for Business Ethics at Bentley University, 115(2): 147-171.

Aubert, N. \& Gaulejac, V. (2007). Le cout de l'excellence. Paris: Seuil.

Barciela, E. (2007). Bases teórico-metodológicas para un enfoque de la gestión de la cultura organizacional en las sedes universitarias municipales. En: Acimed, 16(2)

Bietti, L. (2009). Entre la cognición política y la cognición social: el discurso de la memoria colectiva en Argentina. En: Discurso \& Sociedad, 3(1): 44-89

Biggart, N. \& Beamish, T. (2003). The economic sociology of conventions: habit, custom, practice, and routine in market order. En: Annual Review of Sociology, 29: 443-64.

Bonilla, E. \& Rodríguez, P. (2005). Más allá del dilema de los métodos: la investigación en ciencias sociales. Primera reimpresión. Bogotá: Uniandes.

Bourdieu, P. (1982). Ce que parler veut dire. Paris : Fayard.

Bourdieu, P. (2000) La distinción: criterios y bases sociales del gusto. Madrid: Taurus.

Bourdieu, P. (2001). Las estructuras sociales de la economía. Buenos Aires: Manantial.

Bourdieu, P. (2007). Razones y lecciones de una práctica sociológica. Buenos Aires: Manantial.

Bourdieu, P. \& Wacquant, L. (1995). Respuestas, por una antropología reflexiva. México: Grijalbo.

Calderón, G. (1999). Cultura organizacional: su concepción, sentido y significado. En: Decisión Administrativa, Centro de Publicaciones de la Universidad Nacional de Colombia, Sede Medellín, 2(2): 17 - 26.

De la Garza, E. (2005). Del concepto ampliado del trabajo, al sujeto laboral ampliado. En: De la Garza, E. (comp.) Sindicatos y nuevos movimientos sociales en América Latina. CLACSO.

Emirbayer, M. \& Johnson, V. (2008). Bourdieu and organizational analysis. En: Theor. Soc., 37: 1-44.
Enríquez, Á. (2007). La significación en la cultura. Concepto base para el aprendizaje organizacional. En: Universitas Psychologica, 6: $155-162$.

García, C. (2006) Una aproximación al concepto de cultura organizacional. En: Universitas Psychologica, 5(oo1): 163 - 174.

Gartman, D. (2007). The strength of weak programs in cultural sociology: a critique of Alexander's critique of Bourdieu. En: Theory and Society, 36(5): 381-413.

Goffman, I. (2001). Internados. Ensayos sobre la situación social de los enfermos metales. Amorrortu editores.

Gonzales, M. \& Parra, P. (2008). Caracterización de la cultura organizacional. Clima organizacional, motivación, liderazgo y satisfacción de las pequeñas empresas del Valle de Sugamuxi y su incidencia en el espíritu empresarial. En: Pensamiento \& Gestión, Universidad del Norte, 25: 40-57.

Hallett, T. (2003). Symbolic power and organizational culture. En: Sociological Theory, 21(2): 128-149.

Herzberg, F., Mausner, B. \& Snyderman, B. (1960). The motivation to work. En: The Sociological Quarterly, (1)4: 260-261.

Hodgson, G. (2007). Economía institucional y evolutiva contemporánea. México: Casa abierta al tiempo.

Hodgson, G. (2004). Reclaiming habit for institutional economics. En: Journal of Economic Psychology, 25: 651-660.

Hodgson, G. (1996). The evolution of institutional economics. Agency, structure and darwinism in american institutionalism. London: Routledge.

Hofstede, G. (1999) Culturas y organizaciones. El software mental, la cooperación internacional y su importancia para la supervivencia. Madrid: Alianza Editorial.

Hunt, M. (2010). "Active waiting": habits and the practice of conducting qualitative research. En: International Journal of Qualitative Methods, 9(1): 69-76.

Ignatow, G. (2009). Culture and embodied cognition: moral discourses in internet support groups for overeaters. En: Social Forces, 88(2): 643-670.

Ikefuji, M. \& Mino, K. (2009). Internal vs external habit formation in a growing economy with overlapping generations. Kioto University. Discussion paper $N^{\circ} 676$.

Kondra, A. \& Hurst, D. (2009) Institutional processes of organizational culture. En: Culture and organization, 15(1). Standing Conference on Organizational Symbolism.

Lally, P., Wardle, J. \& Gardner, B. (2011). Experiences of habit formation: a qualitative study. En: Psychology, Health \& Medicine, 16(4): 484-489.

Lastra, R. \& de la Rosa, A. (2006). Las ciencias humanas y el paso de la filogenia a la ontogénesis. En: Acta Universitaria, Universidad de Guanajuato, 16(1): 29-39,

Levin, I. \& Gottlieb, J. (2009) Realigning Organization culture for optimal performance six principles and eight practices. En: Organization Development Journal, 27(4). O. D. Institute

Mangi, L. (2009). Neoinstitutionalism and the appropriation of Bourdieu's work: a critical assessment. En: Rev. adm. Empres., 49(3): 323-336.

Martínez, A. (2007). Pierre Bourdieu: razones y lecciones de una práctica sociológica. Manantial: Buenos Aires.

Mayo, E. (2003). The human problems of an industrial civilization. [Reprint]. Routledge

Montealegre, J. \& Calderón, G. (2007). Relaciones entre actitud hacia el cambio y cultura organizacional. En: INNOVAR. INNOVAR, 17(29): 49-69. 
Nelson, R. \& Winter, S. (2002). Evolutionary theorizing in economics. En: Journal of Economic Perspectives, 16(2): 23-46.

Ojeda, H. (2009). Cultura organizacional y habitus: análisis de un relato etnográfico. Prácticas de oficio. Investigación y reflexión en ciencias sociales, $\mathrm{N}^{\circ} 5$.

Rodríguez, O. (2010) La cultura organizacional: comprensiones, características y reflexiones en una institución de carácter social y comunitario. En: Sotavento, 14: 54-60. Universidad Externado de Colombia.

Sainsaulieu, R. (1997). Sociologie de l'entreprise: organisation, culture et développement. Presses de Sciences Po et Dalloz. Paris.

Sánchez, J., Lanero, A., Yurrebaso, A. \& Tejero, B. (2007). Cultura y desfases culturales de los equipos de trabajo: implicaciones para el compromiso organizacional. En: Psicothema, 19(2): 218-224.

Song, J., Baek, K. \& Chermack, T. (2009). The dimensions of learning organization questionnaire -DLOQ- A validation study in a Korean context. En: Human Resource Development Quarterly, 20(1): 43-64. Wiley Periodicals, Inc.
Strategor (1995). Estrategia, estructura, decisión, identidad. Política general de empresa. Barcelona: Biblioempresa.

Verplanken, B. \& Wood, W. (2006). Interventions to break and create consumer habits. En: Journal Of Public Policy \& Marketing, 25(1): 90-103.

Voronov, M. \& Vince, R. (2012). Integrating emotions into the analysis of institutional work. En: Academy of Management Review, 37(1): 58-81.

Watts, S. (2010). Identification and the cultural organization: how the concept of identification can impact the constituencies who work within cultural organizations. En: The Journal of Arts Management, Law and Society, 40: 200-216.

Zapata, A. \& Rodríguez, A. (2008). Gestión de la cultura organizacional. Bases conceptuales para su implementación. Cali: Universidad del Valle. 